\title{
COMPARISON BETWEEN Mo BIS AND Mo $K$-EDGE ABSORPTION SPECTRUM
}

\author{
E. SoBCZAK \\ Institute of Physics, Polish Academy of Sciences \\ Al. Lotników 32/46, 02-668 Warszawa, Poland
}

The extended X-ray bremsstrahlung isochromat fine structure of bcc Mo for the photon energy $5415 \mathrm{eV}$ is studied and compared with the extended X-ray absorption fine structure at the Mo $K$-edge from the literature. In both spectra a minimum of oscillation amplitude is observed at the momentum 4.2 and $5.3 \AA^{-1}$ for extended X-ray bremsstrahlung isochromat fine structure and extended X-ray absorption fine structure respectively, which is caused by the Ramsauer-Townsend effect at $5.5 \AA^{-1}$ and by beating effect at $3.7 \AA^{-1}$ from the first and second atomic shells. The Fourier transform of Mo extended X-ray bremsstrahlung isochromat fine structure was found to be in good agreement with the radial structure function of bcc Mo only in the case when the integration was performed in momentum range below $4.2 \AA^{-1}$.

PACS numbers: $79.20 . \mathrm{Kz}, 78.70 . \mathrm{Dm}$

Electrons bombarding a solid produce $\mathrm{X}$-ray bremsstrahlung radiation. The bremsstrahlung spectra can be measured either in an isochromat mode or in a constant initial state mode. In this work the bremsstrahlung isochromat spectroscopy (BIS) method is applied. In BIS method an X-ray monochromator is set to pass radiation at a fixed frequency $\nu$. The intensity of bremsstrahlung radiation is recorded as a function of voltage at the $\mathrm{X}$-ray tube.

The bremsstrahlung process can be treated at some conditions as a time-reversed photoelectron effect. There are strong similarities between BIS and X-ray absorption spectra, since in both phenomena the final electron originates in the space close to the nucleus. The transition probabilities for BIS strongly depend on the atomic number and photon energy and transitions to $p$-type states usually dominate over the others $[1,2]$.

The aim of this work was to study the extended X-ray bremsstrahlung isochromat fine structure (EXBIFS) of Mo and to compare it with the extended $X$-ray absorption fine structure (EXAFS) at the Mo $K$-edge. The EXBIFS of some fcc metals, $\mathrm{Cu}, \mathrm{Ni}$, and $\mathrm{Co}$, was already studied and compared with EXAFS over the $K$-edge of these metals [3]. The present work for Mo and another work for $\mathrm{Fe}$ [4] are the first EXBIFS studies for bcc structure. It is shown here and in Ref. [4] 
that studies for bcc structure are more difficult than for fcc structure, because the first and second atomic shells in bcc structure are close to each other.

BIS measurements were performed at the photon energy $5415 \mathrm{eV}$ using a vacuum $\mathrm{X}$-ray bremsstrahlung isochromat spectrometer constructed in the Institute of Physics of P.A.S. A $0.25 \mathrm{~mm}$ thick Mo foil was mounted on a water-cooled target of an X-ray tube. Zeolite sorption pumps and an ion pump kept the vacuum in the $\mathrm{X}$-ray tube at $10^{-4} \mathrm{~Pa}$. The high voltage was stabilized with accuracy $2 \times 10^{-5}$. The high voltage was automatically increased by $0.5 \mathrm{~V}$ steps.

The Johann-type X-ray spectrometer with the Si(220) crystal, bent elastically in a steel holder to a cylindrical curvature of $1 \mathrm{~m}$ radius, was used. The spectrometer calibration was made using the $\mathrm{Cr} K_{\alpha_{1}}$ emission line (the photon energy equal to $5414.72 \mathrm{eV}$ ) from a $\mathrm{Cr}$ plate excited by $7.4 \mathrm{keV}$ electrons. The $\mathrm{Cr} K_{\alpha_{1,2}}$ dublet was fully resolved and the measured FWHM of the $\mathrm{Cr} K_{\alpha_{1}}$ was $3.3 \mathrm{eV}$, while the natural width of this line is $2.07 \mathrm{eV}$. The width of the total spectrometer function FWHM $=1.8 \mathrm{eV}$, including the monochromator window and the energy spread of electrons emitted from the cathode, was estimated from the broadening of the Pd BIS threshold [1]. The advantage of using a proportional counter as a detector was a very low background equal to 16 counts/min.

The BIS of Mo was measured with $0.5 \mathrm{~V}$ steps up to about $150 \mathrm{~V}$ above the threshold. The incident electron beam current, $13 \mathrm{~mA}$, was kept constant over the energy range studied. Several isochromats were measured and added. The power of the X-ray tube was experimentally adjusted to avoid melting of the sample, and, on the other hand, to attain sufficiently high temperature of the surface to preserve from surface contaminations during long-time measurements. No changes, neither in a shape nor intensity, of Mo isochromats were observed during the 40 hours measurements.

The Mo BIS spectrum up to $30 \mathrm{eV}$ above the threshold measured by means of the same apparatus and compared to the theoretical Mo BIS spectrum calculated using partial densities of states has been already reported [5]. It was found that nearly all features of the Mo BIS up to $30 \mathrm{eV}$ are related to p-type electron density of states, except for the first maximum which originates from the $d$-type electron states [5].

The $K$-edge absorption spectrum of Mo was taken from the literature [6]. It was measured at the room temperature using synchrotron radiation. In Fig. 1, the Mo BIS and Mo $K$-edge absorption spectra are shown in energy scale. The zero energy was chosen arbitrarily at the middle point of the BIS threshold. There is no adequate feature in the Mo $K$-edge absorption spectrum, therefore its zero energy was chosen in such a way that the first maximum of absorption relates to the second maximum of BIS intensity. The zero of momentum scale corresponds to the energy value $-7 \mathrm{eV}$ in the above relative energy scale.

A significant difference between the BIS and absorption spectra in the whole energy range is observed. First maximum of BIS has no respective peak in the $K$-edge absorption spectrum (or it is negligibly small), because it is mostly $d$-type. The Mo $K$-edge absorption spectrum is broadened due to the natural width $5 \mathrm{eV}$ of the $K$-level. Such a broadening does not exist in BIS. The oscillations of Mo BIS are very strong near the threshold and become hardly damped at $50 \mathrm{eV}$ 


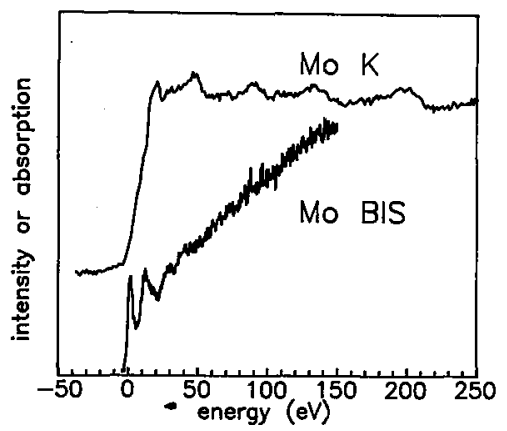

Fig.1

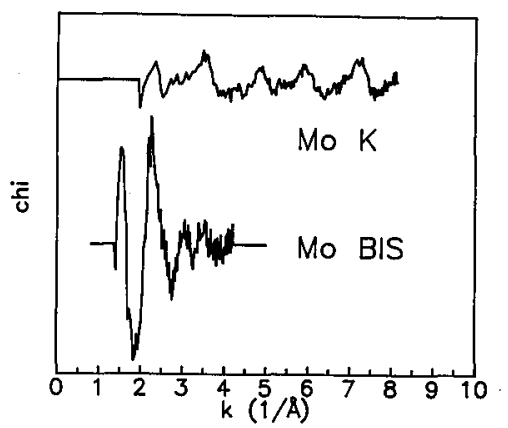

Fig.2

Fig. 1. Mo $K$-edge absorption spectrum (upper curve) by Kostroun et al. [6] and Mo $\mathrm{X}$-ray bremsstrahlung isochromat for the photon energy $5415 \mathrm{eV}$ (lower curve).

Fig. 2. Oscillation functions of Mo $K$-edge absorption spectrum (upper curve) and Mo BIS spectrum (lower curve) as a function of momentum.

above the threshold, because of high temperature of the Mo sample during BIS measurements.

The oscillation function $\chi(k)$ for EXBIFS and EXAFS was found by fitting a polynomial of the third degree. The oscillation functions for EXBIFS and EXAFS are plotted in Fig. 2. The Fourier transforms, calculated using a Hanning window function in the momentum ranges 1.4-4.2 $\AA^{-1}$ and $2.0-8.1 \AA^{-1}$ for EXBIFS and EXAFS respectively, are shown in Fig. 3. The Fourier transform of Mo BIS is in

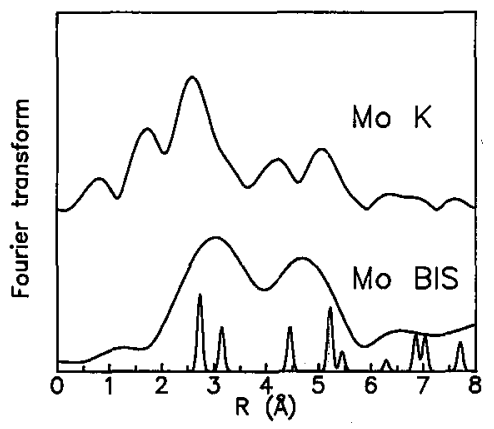

Fig. 3

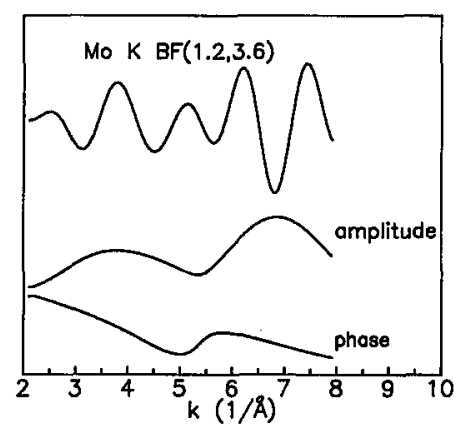

$\mathrm{Fig} .4$

Fig. 3. Moduli of the Fourier transforms for Mo $K$-edge EXAFS (upper curve) and Mo EXBIFS (middle curve) compared with the radial structure function of bcc Mo (lower curve).

Fig. 4. The Fourier backtransform of the Mo $K$-edge EXAFS transform in Fig. 3 performed in the $R$ range 1.2-3.6 $\AA$ : the real part (upper curve), the amplitude (middle curve) and the phase shift (lower curve). 
good agreement with the radial structure function of bcc Mo, which is shown in the same figure.

The first- and second-shell atomic peaks cannot be separated in the Fourier transform because of the small intershell distance in the bcc structure, which equals $0.422 \AA$ for the bcc Mo. However, quite a big splitting of the main peak is observed in the Fourier transform of the Mo $K$-edge EXAFS (Fig. 3). Also similar splitting was observed for the Fourier transform of Mo EXBIFS if integrated in a wider momentum range 1.4-6.3 $\AA^{-1}$. Such a splitting, observed for EXAFS of Ag and heavier elements, is caused by the Ramsauer-Townsend effect, which originates from the fact that the backscattering amplitude has a local minimum and the backscattering phase shift has a phase jump at a momentum value characteristic of each element. The Ramsauer-Townsend effect for Mo should appear at the momentum $5.5 \AA^{-1}$ [7].

The Fourier backtransform of the Mo $K$-edge EXAFS transform from the Fig. 3 was performed in the $R$ range 1.2-3.6 $\AA$ (Fig. 4). The amplitude and the phase shift of oscillations change rapidly their values for the momentum about $5.5 \AA^{-1}$. Similar analysis performed for Mo EXBIFS gave $4.2 \AA^{-1}$ as the value of momentum at which the amplitude and the phase change rapidly. Another effect is a beating of oscillations due to a small separation of atomic shells in Mo, which should cause a local minimum of amplitude and a phase jump at $3.7 \AA^{-1}$ for both spectra. However, these two effects are mixed and therefore a quantitative determination of intershell distance between first and second shells in bcc Mo using a beat method like in bcc Fe [4] is impossible.

The main result of our work is that the extended structure of the Mo BIS is clearly visible and can be analysed by the Fourier transform. It is found that all BIS features except the first maximum of Mo BIS are related to the Mo $K$-edge EXAFS features. In both spectra a minimum of oscillation amplitude is observed at the momentum 4.2 and $5.3 \AA^{-1}$ for EXBIFS and EXAFS respectively, which is caused by the Ramsauer-Townsend effect at $5.5 \AA^{-1}$ and by beating effect at $3.7 \AA^{-1}$ from the first and second atomic shells. The Fourier transform of Mo EXBIFS was found to be in good agreement with the radial structure function of bcc Mo only in the case when the integration was performed in the momentum range below $4.2 \AA^{-1}$.

\section{References}

[1] E. Sobczak, J. Auleytner, Phys. Rev. B 37, 6251 (1988).

[2] A. Simůnek, J. Vackáŕ, E. Sobczak, Phys. Rev. B 38, 8515 (1988).

[3] E. Sobczak, J. Auleytner, S. Mobilio, A. Balerna, O. Smotlacha, Physica B 158, 553 (1989).

[4] E. Sobczak, S. Mobilio, Acta Phys. Pol. A 82, 333 (1992).

[5] M.T. Czyżyk, K. Ławniczak-Jabłońska, S. Mobilio, Phys. Rev. B 45, 1581 (1992).

[6] V.O. Kostroun, R.W. Fairchild, C.A. Kukkonen, J.W. Wilkins, Phys. Rev. 13, 3268 (1976).

[7] A.G. McKale, B.W. Veal, A.P. Paulikas, S.-K. Chan, G.S. Knapp, J. Am. Chem. Soc. 110, 3763 (1988). 